\title{
Osteogenesis Capability and Degradation Property Evaluation of Injectable Biomaterials: Comparison of Computed Tomography and Ultrasound
}

\author{
Yan Chen, ${ }^{1}$ Yuting Yan, ${ }^{2}$ Xiaoming Li, ${ }^{3} \mathrm{He} \mathrm{Li},{ }^{2}$ Yue Yuan, ${ }^{2}$ Xiaoying Gao, ${ }^{2}$ Xixi Wu, \\ Jisheng Zhong, ${ }^{2}$ Bomiao Lin, ${ }^{4}$ Yubo Fan, ${ }^{3}$ and Bo $\mathrm{Yu}^{5}$ \\ ${ }^{1}$ Department of Ultrasonic Diagnosis, Zhujiang Hospital of Southern Medical University, Guangzhou 510282, China \\ ${ }^{2}$ The Second Clinical Medical College of Southern Medical University, Guangzhou 510282, China \\ ${ }^{3}$ Key Laboratory for Biomechanics and Mechanobiology of Ministry of Education, School of Biological Science and Medical Engineering, \\ Beihang University, Beijing 100191, China \\ ${ }^{4}$ Department of Radiology, Zhujiang Hospital of Southern Medical University, Guangzhou 510282, China \\ ${ }^{5}$ Department of Orthopedics, Zhujiang Hospital of Southern Medical University, Guangzhou 510282, China
}

Correspondence should be addressed to Xiaoming Li; x.m.li@hotmail.com and Bo Yu; gzyubo@gmail.com

Received 19 May 2013; Accepted 17 June 2013

Academic Editor: Shuming Zhang

Copyright (C) 2013 Yan Chen et al. This is an open access article distributed under the Creative Commons Attribution License, which permits unrestricted use, distribution, and reproduction in any medium, provided the original work is properly cited.

\begin{abstract}
Injectable biomaterials, which can be physically inserted into a target site without the use of surgery, have received increasing attention in tissue engineering during the last decade. There is also a growing need for quantitative evaluation of the injectable biomaterial directly and noninvasively. The objectives of this study are to originate a quantitative noninvasive technique for evaluation of in situ forming bone biomaterials and to validate the feasibility of diagnostic ultrasound images analysis technique. The potential of ultrasound for quantitative evaluation of tissue development was compared with computed tomography (CT) in vivo. A strong correlation was witnessed between ultrasound gray-scale values $(\mathrm{GV})$ and volumetric mean of CT value $\left(\mathrm{HU}_{m}\right)(r=0.95)$. Meanwhile, the volume of the material area could be estimated by ultrasound maximum cross-section pixel, which demonstrates a certain consistency with CT mask volume in 3D reconstruction images $(r=0.87)$. In conclusion, ultrasound imaging, which is corresponding with the traditional CT, can be used to evaluate osteogenesis capability and degradation property of injectable biomaterials. It may be a noninvasive, nonradioactive, and effective aid to monitor ossification and reconstruction of biomaterials at the implant region for bone defect repair.
\end{abstract}

\section{Introduction}

An injectable thermosensitive drug-delivery hydrogel was reported by NATURE in 1997 and was demonstrated biodegradable, biocompatible, and nontoxic by implantation experiments [1]. Since then, biomaterials and tissue engineering have aroused high academic attention and become a hotspot in interdisciplinary research. Hydrogel biomaterials, with prominent superiority, undoubtedly provide a new insight into the bone reconstruction field [2-5]. Recently, the feasibility and efficacy of hyaluronic acid-g-chitosang-poly (N-isopropyl acrylamide) (HA-CPN) copolymer, chitosan/gelatin/glycerophosphate (C/G/GP) hydrogel, and chitosan/collagen/ $\beta$-glycerophosphate (GP) hydrogel were confirmed in dog models of congenital cleft palate, rabbit models of articular cartilage defects, and rat models of unicortical femoral defects [6-11]. By combining the chitosan (CS), nanohydroxyapatite (nHA), and collagen (Col), an in situ forming hydrogel biomaterial was fabricated by using a biomimetic strategy $[12,13]$.

The evaluation of composite development in vivo has been a key issue in the research of in-situ forming biomaterials. Nowadays, biomaterials can be evaluated by several methods. Histology, general morphology, X-ray, and immunohistochemistry are universally and commonly used in the evaluation of bone tissue engineering through 
in vivo studies [14]. These traditional high-sensitivity methods failed to detect transformation noninvasively in real time always at the cost of experiment-animal sacrifice. Callus in $\mathrm{X}$-ray images obviously lags behind the actual time, a visual resolution just with $25 \%$ discrepancy of bone mineral content. The traditional methods portray little value in quantitative and noninvasive system of injectable gel evaluation. Present applications of noninvasive and quantitative bone tissue engineering assessment include dual-energy X-ray absorptiometry (DXA) and quantitative computed tomography (QCT). The result of DXA, areal bone mineral density (BMD) $\left(\mathrm{g} / \mathrm{cm}^{2}\right)$, ignores the bone's spatial geometric distribution to a large extent, which is limited by its two-dimensionality $[15,16]$. Volumetric BMD can be measured only by QCT now, and its $3 \mathrm{D}$ images can be used to accurately evaluate the size, geometry and density of both cortical and trabecular bones [17]. QCT, which improves the performance of DXA, however, has similar disadvantage as DXA and increases the risk of high radiation. It is limited in clinic due to its high cost, high radiation, requirement for precise apparatus, and limited access to CT scanners. Some other methods such as micro-CT $(\mu \mathrm{CT})$, magnetic resonance imaging (MRI), and impulse response method have been tentatively but limitedly applied in bone density determination.

In the last decade, quantitative ultrasound (QUS) has been used to assess the fracture risk of osteoporosis. Ultrasonic parameters then developed to be an ingenious aid to reflect elasticity and mechanical intensity of biomaterials [1820]. In our previous study, we found that diagnostic ultrasound could be used for evaluation of injectable biomaterials [13]. However, the correlations between CT and diagnostic ultrasound in the evaluation of the biomaterials were not clear. So in this paper, we quantitatively compared computed tomography (CT) with ultrasound in the evaluation of injectable hydrogel biomaterials.

\section{Materials and Methods}

2.1. Preparation of Composites. CS and CS/nHAC were prepared according to $[12,13]$. First, nanohydroxyapatite/collagen (nHAC) provided by Tsinghua University was sterilized by $\gamma$-ray irradiation $(1.5 \mathrm{Mrad})$. The complex solution which dissolved CS [2 g, from Shandong A K Biotech Ltd (China)] in a hydrochloric acid solution $(98 \mathrm{~mL}$, $0.1 \mathrm{M})$ underwent high-pressure disinfection $\left(1.22 \mathrm{kPa}, 120^{\circ} \mathrm{C}\right.$, $20 \mathrm{~min})$, and after addition of $1: 1 \mathrm{nHAC}(0.02 \mathrm{~g} / \mathrm{mL})$, the complex solution was adjusted to $\mathrm{pH} 7.0$ by adding GP $\left(\mathrm{C}_{3} \mathrm{H}_{7} \mathrm{Na}_{2} \mathrm{O}_{6} \mathrm{P}\right.$, from USA Sigma). After standing in a $37^{\circ} \mathrm{C}$ thermostat water bath for 5-10 $\mathrm{min}, \mathrm{CS} / \mathrm{nHAC}$ was prepared. CS was prepared in the same procedure. All the operations strictly followed aseptic technique in an aseptic manipulation cabinet.

2.2. Implantation Experiments in Rats. Ten female Wistar rats (250-300 g, average $280 \mathrm{~g}$ ), which were supplied by the Animal Research Center at Guangdong Province, China, were randomly divided into two groups. After inhalation anesthesia using $1 \%$ isoflurane, CS and CS/nHAC were injected into the two groups in the subcutaneous dorsum, respectively, by a 26-gauge needle attached to a $2 \mathrm{~mL}$ syringe. All the animals were operated under the mediation of diagnostic ultrasound. The needle, syringe, and injectable hydrogels were kept in a medical refrigerator before injection. All the operations followed an agreement approved by the Institutional Animal Care and Use Committee at Southern Medical University.

2.3. Ultrasound Imaging Analysis. The implants were examined using an ALOKA prosound $\alpha-10$ premier diagnostic ultrasound system with a $12 \mathrm{MHz}$ transducer, 55 dynamic range, 1.1 mechanical index, and 80 transmission gain at the 1 st hour (1 h), 14th day (14 d), and 28th day (28 d) after implantation. The images were transferred to an off-line computer (Q-lab, Philips Medical Systems) for the measurement of gray-scale value $(\mathrm{GV})$.

The GVs of ultrasound images were demonstrated to be related to the stiffness of the tissue [13]. That is, there may be a positive correlation between osteogenesis of a composite and the backscattered signal caused by the difference in mechanical impedance, which was displayed as a GV ranging from 0 to 256. In addition, the number of pixels of a region-of-interest (ROI) can indirectly reflect the implant's cross-sectional area. Consequently, the GVs and the pixel data of the implants for each group at three time points ( $1 \mathrm{~h}, 14 \mathrm{~d}$, and $28 \mathrm{~d}$ ) were obtained from 5 samples. All image analyses were performed on MATLAB 7.0.

2.4. CT Imaging Analysis. CT was performed using an Elscint Exel 2400 elite CT scanner, with the exposure parameters of $120 \mathrm{kV}$ and $15 \mathrm{~mA}$. The air-filled external ear canals were used as landmarks to position a single transverse $2.5 \mathrm{~mm}$ slice. The scan images for the two groups at $1 \mathrm{~h}, 14 \mathrm{~d}$, and $28 \mathrm{~d}$ were exported to DICOM 3.0 format and were imported into the imaging software Mimics. In Mimics, an ROI was generated, and the CT value (HU), the voxel, and the volume were recorded at each phantom density site. Then, to compare degradation property evaluation with medical diagnostic ultrasound, 3D volumetric reconstructions of the implants were performed using the $3 \mathrm{D}$ calculation function. All image analyses were performed on Mimics 10.1.

2.5. Statistical Analysis. Bivariate correlation analysis was adopted to analyze the relationships between CT value and ultrasound GV and between CT mask volume and ultrasound cross-section area. Pearson correlation coefficients were calculated to assess the correlation extent between the two imaging techniques. The four variables (CT value, GV, volume, and cross-section area) for the animals which received CS were compared with those which received CS/nHAC by using Mann-Whitney $U$ test. Flag significant correlations by two-tail test at level 0.05 were considered as a significant difference. Statistical analysis was performed on SPSS 19.0.

\section{Results and Discussion}

3.1. Evaluation of In Situ Forming Bone Biomaterial. For biomaterials bone regeneration, the key point is to balance 

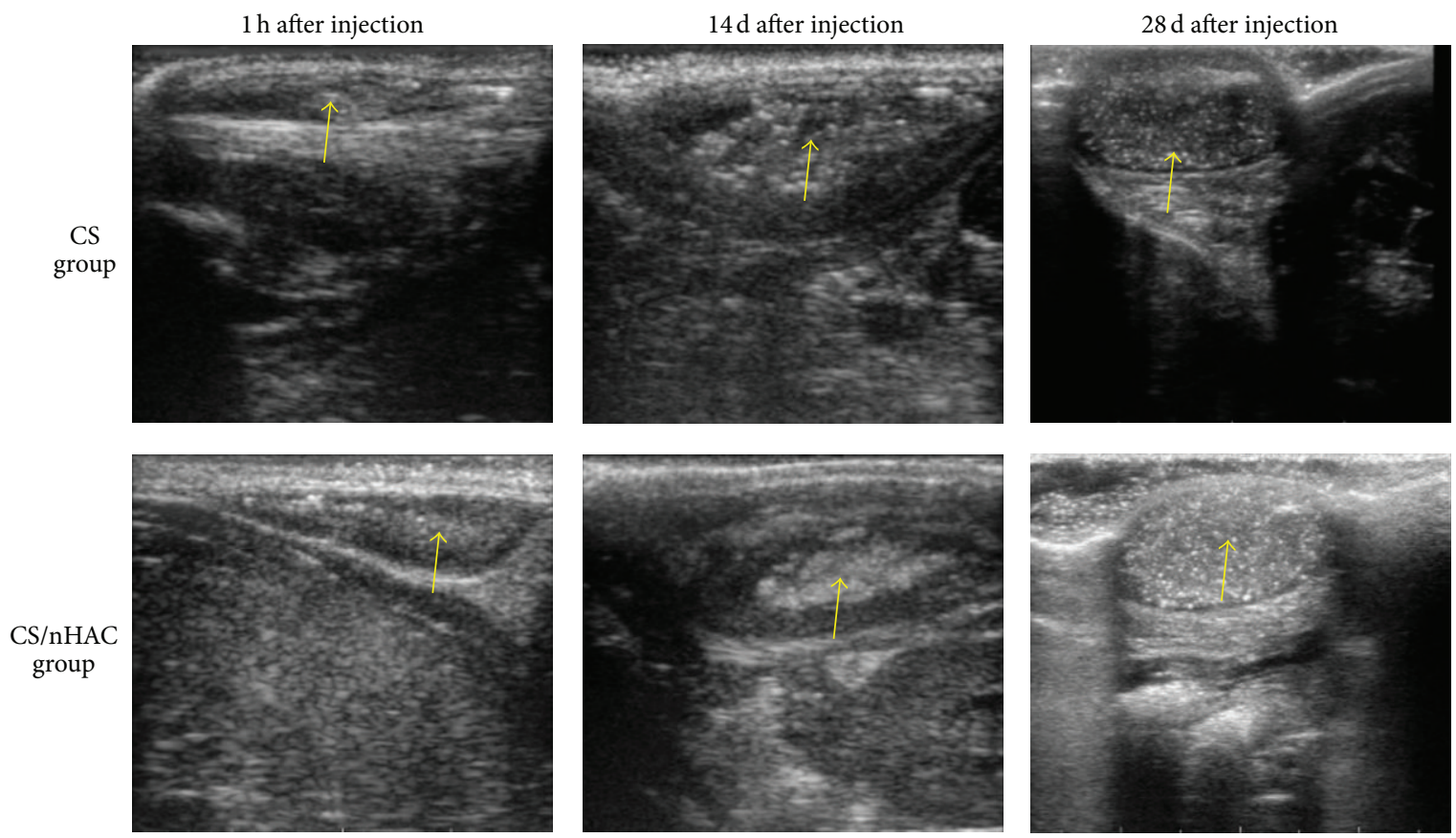

FIGURE 1: Ultrasound images of implantation area, showing the bone regeneration by biomaterials (CS, CS/nHAC) at $1 \mathrm{~h}, 14 \mathrm{~d}$, and $28 \mathrm{~d}$ (the outline of implant is legible, and arrows point to the implantation area in each group and at each time point).

between bone reconstruction and implant degradation. With the proceeding of porosis, the biomaterials self-degrade to accommodate cells, growth factors, and nutriments, and then new bone occupies the space of the implant, so the bone is reconstructed. Yet the transition from basic research to clinical application is limited by the primary weak link about noninvasive and accurate evaluation of the implant's in vivo development. Admittedly, a direct nondestructive quantitative evaluation system is desperately required at the moment.

3.1.1. Osteogenesis Capability. BMD is well accepted as an indicator of osteogenesis capability in biomaterial implant site. Recently, there has been increasing interest in the use of ultrasound for BMD evaluation since it has been first reported to monitor osteoporosis in 1984 [21]. QUS is a promising and relatively new technology for testing BMD by adopting broadband ultrasound attenuation (BUA) and ultrasonic speed of sound (SOS). According to clinical research, QUS can reflect other bone structural characteristics such as microarchitecture, elasticity, and fragility [22]. Supported by the theories of QUS, ultrasonic wave will be largely attenuated through cancellous bone, which is suitable for ultrasonic penetration inspection. Ultrasound, which is real time, dynamic, convenient, inexpensive, noninvasive, nonradiative, and multifaceted, has been widely used to evaluate the characteristics of articular cartilage and elastic tissues and is tentatively applied in in vivo studies in tissue engineering [23]. GV with a high consistency with hydroxyproline is a crucial parameter in diagnostic ultrasound [24]. GV gives an index to acoustic impedance calculated by medium density and sound velocity. Coincidentally, the implantation area of ectopic osteogenesis is just in muscular pouch superficially to the benefit of ultrasonic penetration. Thus, GV could relatively well represent the density of shallow implant materials. In addition, the finally recorded GV somewhat conforms to the microstructure, elasticity, and brittleness in implantation area. In this study, we attempted to evaluate osteogenesis capability by diagnostic ultrasound and to record GVs at $1 \mathrm{~h}, 14 \mathrm{~d}$, and $28 \mathrm{~d}$. The outline of implantation area is distinct due to the different acoustic impedances from biomaterial to surrounding tissues. An increasing intensity of the ultrasound images with time was manifested by GV, which may demonstrate the production of bone matrix and callus (Figure 1). The CS/nHAC group had a mean GV of 32.58 at $1 \mathrm{~h}$, which increased to 120.36 at $28 \mathrm{~d}$ (Table 1).

To verify the feasibility and veracity of ultraphonic assessment, imaging technique based on CT 3D reconstruction was adopted for osteogenesis capability evaluation (Figure 2(a)). According to the principle of QCT, routine CT images were analyzed to quickly and accurately measure the bone density in any section and any form of the implantation area. Computed by $3 \mathrm{D}$ clipping, the volumetric mean of CT value $\left(\mathrm{HU}_{m}\right)$ was defined to describe bone mass in the implantation site. The $\mathrm{HU}_{m}$ of the biomaterial was calculated by $\mathrm{CT}$ value of ROI voxels $\left(\mathrm{HU}_{i}\right)$ and count of ROI voxels $(N)$ as follows:

$$
\mathrm{HU}_{m}=\sum_{i=1}^{N} \mathrm{HU}_{i} / N
$$

A marked regression relationship was confirmed between $\mathrm{HU}_{m}$ and $\mathrm{BMD}$ [25], so $\mathrm{HU}_{m}$ was used to evaluate the osteogenesis capability of biomaterials and thereby to validate 
TABLE 1: ROI GV and cross-section area (behalf of pixel) of ultrasound images. GV is in an uptrend while the section area is in a downtrend with time. The data are expressed as mean \pm standard deviation (SD).

\begin{tabular}{|c|c|c|c|c|c|c|c|c|c|c|c|c|}
\hline \multirow{3}{*}{$\begin{array}{l}\text { No. of } \\
\text { animals }\end{array}$} & \multicolumn{6}{|c|}{ CS group } & \multicolumn{6}{|c|}{ CS/nHAC group } \\
\hline & \multicolumn{2}{|c|}{$1 \mathrm{~h}$} & \multicolumn{2}{|c|}{$14 \mathrm{~d}$} & \multicolumn{2}{|c|}{$28 \mathrm{~d}$} & \multicolumn{2}{|c|}{$1 \mathrm{~h}$} & \multicolumn{2}{|c|}{$14 \mathrm{~d}$} & \multicolumn{2}{|c|}{$28 \mathrm{~d}$} \\
\hline & GV & $\begin{array}{c}\text { Section } \\
\text { area }\end{array}$ & GV & $\begin{array}{c}\text { Section } \\
\text { area }\end{array}$ & GV & $\begin{array}{c}\text { Section } \\
\text { area }\end{array}$ & GV & $\begin{array}{c}\text { Section } \\
\text { area }\end{array}$ & GV & $\begin{array}{c}\text { Section } \\
\text { area }\end{array}$ & GV & $\begin{array}{c}\text { Section } \\
\text { area }\end{array}$ \\
\hline 1 & 32.46 & 21657 & 51.48 & 18827 & 56.12 & 7098 & 39.59 & 25467 & 63.39 & 18322 & 129.82 & 14727 \\
\hline 2 & 36.08 & 20233 & 49.77 & 10951 & 59.54 & 8934 & 27.73 & 21930 & 53.40 & 14681 & 135.80 & 13400 \\
\hline 3 & 32.23 & 31197 & 40.69 & 17027 & 65.59 & 9526 & 32.07 & 20422 & 51.89 & 15498 & 103.28 & 12672 \\
\hline 4 & 30.75 & 24518 & 49.03 & 13932 & 52.71 & 7321 & 28.81 & 22797 & 48.26 & 15029 & 94.96 & 14309 \\
\hline 5 & 29.93 & 28072 & 39.84 & 12385 & 68.47 & 5997 & 34.68 & 23733 & 60.25 & 14792 & 137.94 & 10582 \\
\hline $\bar{X} \pm s$ & $\begin{array}{c}32.29 \pm \\
2.4\end{array}$ & $\begin{array}{c}25135.4 \pm \\
4524\end{array}$ & $\begin{array}{c}46.16 \pm \\
5.5\end{array}$ & $\begin{array}{c}14624.4 \pm \\
3257\end{array}$ & $\begin{array}{c}60.49 \pm \\
6.5\end{array}$ & $\begin{array}{c}7775.3 \pm \\
1435\end{array}$ & $\begin{array}{c}32.58 \pm \\
4.8\end{array}$ & $\begin{array}{c}22869.8 \pm \\
1895\end{array}$ & $\begin{array}{c}55.44 \pm \\
6.2\end{array}$ & $\begin{array}{c}15664.4 \pm \\
1518\end{array}$ & $\begin{array}{c}120.36 \pm \\
19.8\end{array}$ & $\begin{array}{c}13138.0 \pm \\
1637\end{array}$ \\
\hline
\end{tabular}

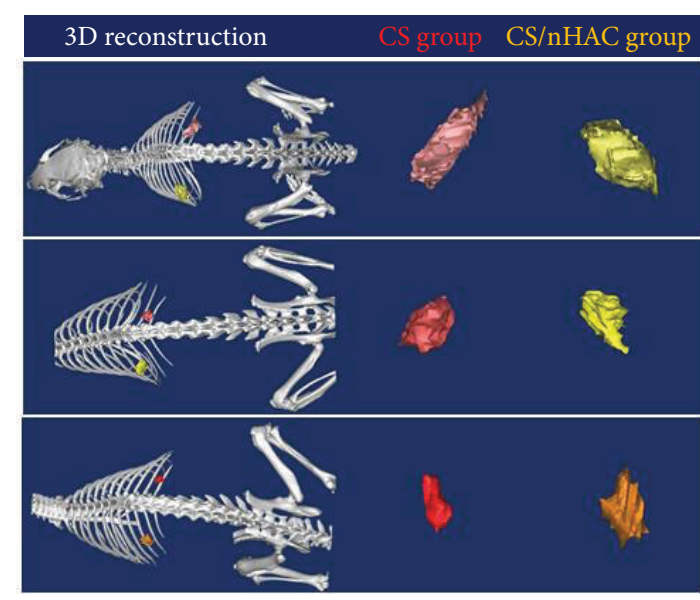

(a)

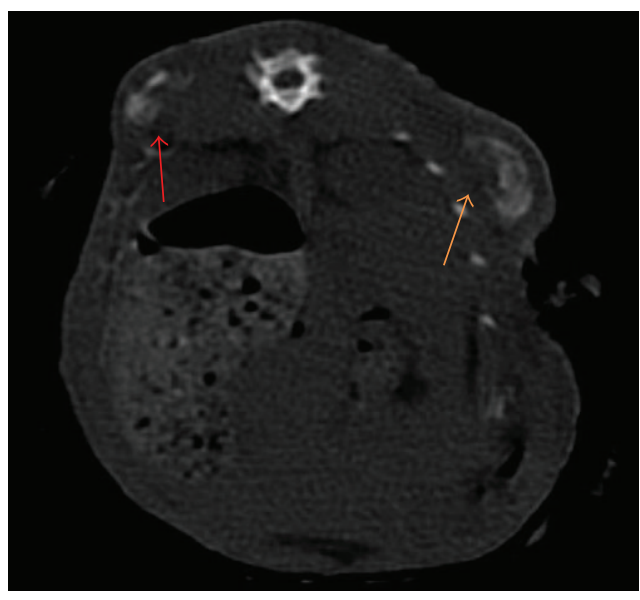

(b)

FIGURE 2: The CT images of implant. (a) 3D reconstruction of ROI (implants). The left sights reflect the positions of implants in rats, and the right sights reflect the shape of implants (the color depth roughly reflects the CT value). The CT values are in an uptrend while the volume of implants is in a downtrend. (b) The CT images at the 28th day. The outline of implants is not as clear as ultrasound image (red arrow points to the CS Group and yellow arrow to the CS/nHAC group).

the feasibility of ultrasonic assessment system. The CT images prominently demonstrated bright zone in implantation area (Figure 2(b)). $\mathrm{HU}_{m}$ remarkably increases with time in implantation area (Table 2).

3.1.2. Degradation Property. It is a common view that an ideal bone biomaterial should have not only a high osteogenesis capability but also biodegradability, which can reduce the number of necessary operations and shorten the recovery time for patients [26-28]. An ideal biomaterial also will not degrade rapidly and unpredictably and may maintain function for the full healing and regeneration period of 4-6 months [29]. Then the biomaterial would show sufficient mechanical properties and not collapse during the patient's normal activities. However, few methods are available for evaluating the degradation property of biomaterial, and the animals normally should be sacrificed. Due to the clarity of ROIs in ultrasound images (Figure 1), the maximum cross-section area of ROI could be an index for measuring degradation property. For corroboration, mask volume of implants in CT, which was calculated by count of ROI voxels, was also recorded in $3 \mathrm{D}$ reconstruction to explore biomaterial degradation in vivo, and the implantation volume showed an obvious downtrend (Figure 2(a) and Table 2).

\subsection{The Correlation between Ultrasound and CT Parameters.} Identifying the parameters for osteogenesis capability and degradation property is a complex and challenging assignment in tissue engineering and regenerative medicine. CT, which accounts for some inherent characteristics of bone biomaterials (bone mass, size, and density), is well accepted in the academic field, but its multitudinousness limits CT from wide application. Accordingly, there is a need to identify additional parameters, which can be easily measured in clinic and have superior precision and sensitivity. We focused on diagnostic ultrasound. In order to test its feasibility, a regression analysis between ultrasound parameters and conventional CT parameters was performed (Figure 3 ). The results showed a strong positive correlation between $\mathrm{HU}_{m}$ and ultrasound $\mathrm{GV}$ in either group, $r_{(\mathrm{CS})}=0.967$ and $r_{(\mathrm{CS} / \mathrm{nHAC})}=0.949$, 
TABLE 2: CT value $\left(\mathrm{HU}_{m}\right)$ and mask volume (calculated from voxel) of the implants. $\mathrm{HU}_{m}$ tendency, on the uptrend, is similar as ultrasound $\mathrm{GV}$, while the mask volume is in a downtrend with time. The data are expressed as mean $\pm \mathrm{SD}$.

\begin{tabular}{|c|c|c|c|c|c|c|c|c|c|c|c|c|}
\hline \multirow{3}{*}{$\begin{array}{l}\text { No. of } \\
\text { animals }\end{array}$} & \multicolumn{6}{|c|}{ CS group } & \multicolumn{6}{|c|}{ CS/nHAC group } \\
\hline & \multicolumn{2}{|c|}{$1 \mathrm{~h}$} & \multicolumn{2}{|c|}{$14 \mathrm{~d}$} & \multicolumn{2}{|c|}{$28 \mathrm{~d}$} & \multicolumn{2}{|c|}{$1 \mathrm{~h}$} & \multicolumn{2}{|c|}{$14 \mathrm{~d}$} & \multicolumn{2}{|c|}{$28 \mathrm{~d}$} \\
\hline & $\mathrm{HU}_{m}$ & $\begin{array}{l}\text { Volume } \\
\left(\mathrm{mm}^{3}\right)\end{array}$ & $\mathrm{HU}_{m}$ & $\begin{array}{l}\text { Volume } \\
\left(\mathrm{mm}^{3}\right)\end{array}$ & $\mathrm{HU}_{m}$ & $\begin{array}{l}\text { Volume } \\
\left(\mathrm{mm}^{3}\right)\end{array}$ & $\mathrm{HU}_{m}$ & $\begin{array}{l}\text { Volume } \\
\left(\mathrm{mm}^{3}\right)\end{array}$ & $\mathrm{HU}_{m}$ & $\begin{array}{l}\text { Volume } \\
\left(\mathrm{mm}^{3}\right)\end{array}$ & $\mathrm{HU}_{m}$ & $\begin{array}{l}\text { Volume } \\
\left(\mathrm{mm}^{3}\right)\end{array}$ \\
\hline 1 & 88.91 & 341.89 & 132.15 & 184.71 & 189.52 & 115.89 & 92.46 & 301.93 & 156.62 & 249.82 & 341.65 & 185.28 \\
\hline 2 & 90.59 & 332.10 & 136.65 & 179.91 & 185.78 & 130.06 & 85.59 & 382.84 & 164.78 & 235.77 & 294.87 & 168.08 \\
\hline 3 & 94.23 & 330.02 & 129.47 & 175.65 & 198.00 & 123.78 & 87.43 & 291.54 & 148.23 & 241.04 & 325.42 & 177.32 \\
\hline 4 & 83.53 & 349.74 & 139.34 & 180.75 & 173.93 & 103.00 & 91.58 & 351.88 & 154.30 & 232.93 & 380.77 & 195.70 \\
\hline 5 & 88.09 & 314.80 & 124.04 & 182.86 & 206.42 & 117.25 & 88.24 & 332.25 & 162.59 & 261.60 & 354.67 & 192.58 \\
\hline $\bar{X} \pm s$ & $\begin{array}{c}89.07 \pm \\
3.9\end{array}$ & $\begin{array}{c}333.71 \pm \\
13.2\end{array}$ & $\begin{array}{c}132.33 \pm \\
6.0\end{array}$ & $\begin{array}{c}180.78 \pm \\
3.4\end{array}$ & $\begin{array}{c}190.73 \pm \\
12.3\end{array}$ & $\begin{array}{c}117.00 \pm \\
10.1\end{array}$ & $\begin{array}{c}89.06 \pm \\
2.9\end{array}$ & $\begin{array}{c}332.09 \pm \\
37.2\end{array}$ & $\begin{array}{c}157.30 \pm \\
6.6\end{array}$ & $\begin{array}{c}244.23 \pm \\
11.6\end{array}$ & $\begin{array}{c}339.48 \pm \\
32.1\end{array}$ & $\begin{array}{c}183.79 \pm \\
11.3\end{array}$ \\
\hline
\end{tabular}

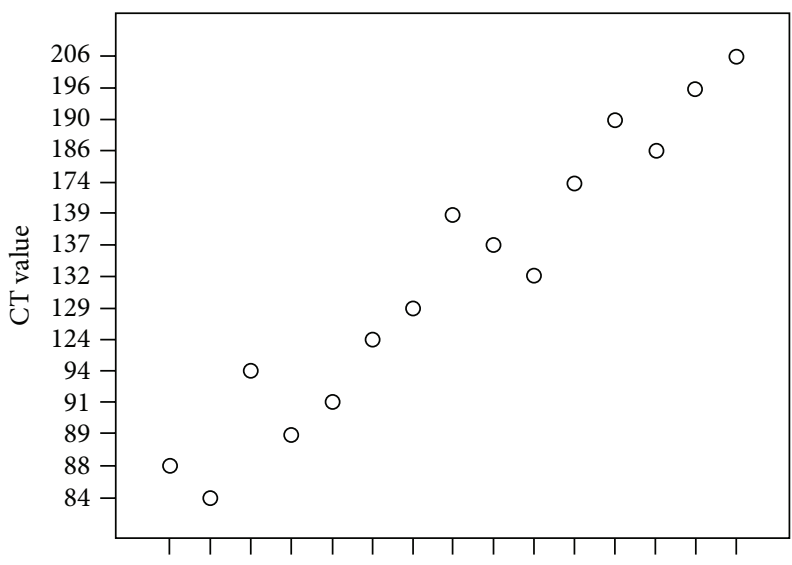

$\begin{array}{lllllllllllllll}30 & 31 & 32 & 32 & 36 & 40 & 41 & 49 & 50 & 51 & 53 & 56 & 60 & 66 & 68\end{array}$ Ultrasound GV

(a)

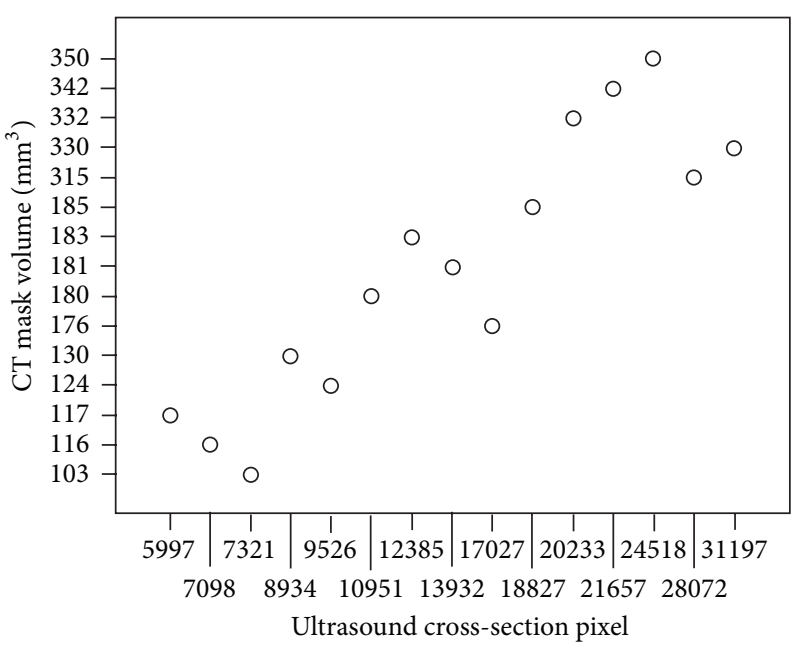

(c)

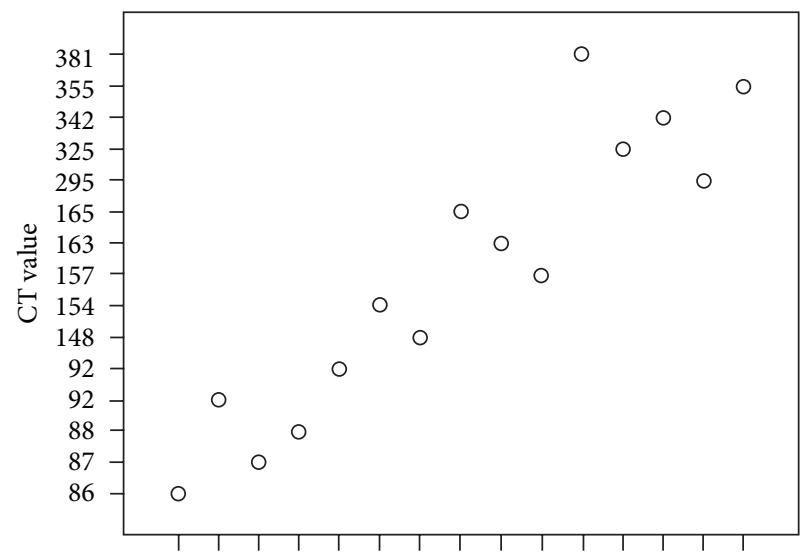

$\begin{array}{llllllllllll}28 & 29 & 32 & 35 & 40 & 48 & 52 & 53 & 60 & 63 & 95 & 103130136138\end{array}$ Ultrasound GV

(b)

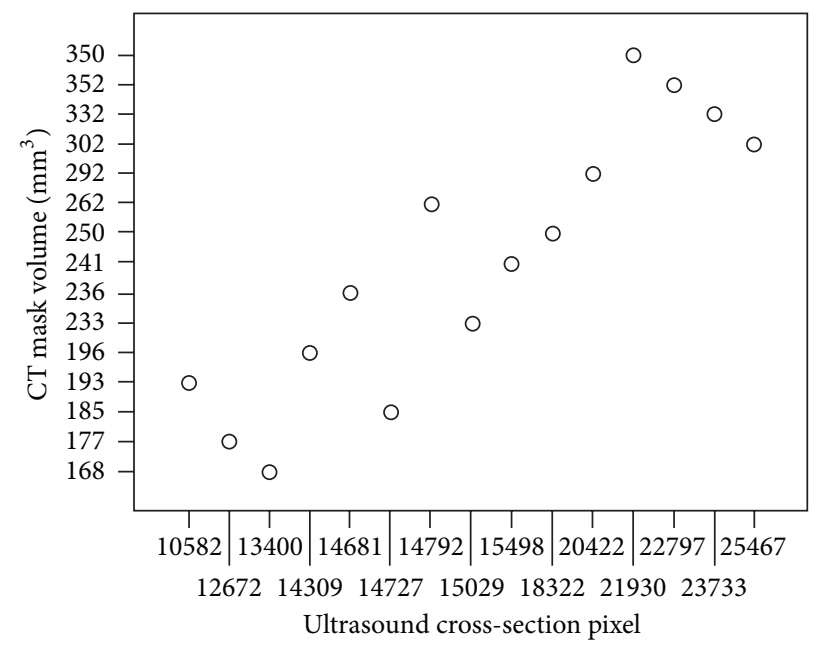

(d)

FIGURE 3: The scatter diagrams in comparison of CT value and ultrasound GV, CT volume, and pixel size. Ultrasound GVs for to abscissa and CT values for ordinate in the CS group (a) and the CS/nHAC group (b); ultrasound section pixel to abscissa and CT mask volume to ordinate in the CS group (c) and the CS/nHAC group (d); the positive correlations between CT value and GV and between CT volume and pixel size are obvious. 
TABLE 3: The correlation coefficients between CT value and ultrasound GV and between CT mask volume and ultrasound section pixel in the CS and the CS/nHAC groups. High correlation with low $P$ value demonstrates that the property evaluation is analogue between ultrasound and CT.

\begin{tabular}{lcc}
\hline & $\begin{array}{cc}\mathrm{CT} \mathrm{HU}_{m} \text { and ultrasound } \\
\mathrm{GV}\end{array}$ & $\begin{array}{c}\text { CT mask volume and } \\
\text { ultrasound cross-section } \\
\text { pixel }\end{array}$ \\
\hline $\mathrm{CS}$ & $r=0.967, P=0.001$ & $r=0.906, P=0.023$ \\
$\mathrm{CS} / \mathrm{nHAC}$ & $r=0.949, P=0.000$ & $r=0.871, P=0.001$ \\
\hline
\end{tabular}

indicating that GV appropriately represents volume density and is a good parameter for assessing osteogenesis capability of biomaterials. As to the degradation property, ultrasound cross-section area is in certain consistency with CT mask volume in either group, $r_{(\mathrm{CS})}=0.906$ and $r_{(\mathrm{CS} / \mathrm{nHAC})}=0.871$. The Pearson correlation coefficient was not as high as that between $\mathrm{HU}_{m}$ and $\mathrm{GV}$, probably due to the irregular shape of the implant. Nevertheless, the correlation coefficient was also over 0.8 , which means that ultrasound cross section area could be used to assess degradation property as an alternative method (Table 3).

3.3. The Two Imaging Techniques for CS and CS/nHAC Assessment. The result of ultrasound showed a high correlation with CT. Then the novel method was used to compare the properties of CS and CS/nHAC and thereby to explore an ideal biomaterial for bone tissue engineering. The results showed that CS/nHAC biomaterial had higher osteogenesis capability and much higher stable degradation rate than CS biomaterial, which were beneficial for later sclerotic implant and bone defect rehabilitation (Figure 4). In the $\mathrm{CS} / \mathrm{nHAC}$ group, the percentage variations of bone mass at $1 \mathrm{~h}$ to $24 \mathrm{~d}$ after injection are $381.18 \%$ and $368.43 \%$ for CT and ultrasound, respectively, which were both apparently higher than those in the CS group. The residual proportions of CS/nHAC at $24 \mathrm{~d}$ are $55.34 \%$ and $57.45 \%$ for CT and ultrasound, respectively, which were also higher than those of the CS group (Table 4). These results demonstrated that the CS/nHAC biomaterial was much superior over the CS biomaterial in bone repair and also verify the high consistency between the two imaging techniques in evaluation of injectable biomaterials.

Actually, ultrasound could provide some information on bone fragility, structure, elasticity, and porosity that cannot be provided by CT. In terms of material biomechanics, ultrasound evaluation may be more credible than bone mass measurement and becomes growingly popular due to its non radialization and convenience. For biomaterial evaluation in bone tissue engineering, animals should be anesthetized to decrease the artifacts when CT is used. On the contrary, animals show higher compliance during ultrasound examination, and the apparatus can be easily operated with a low space requirement and certain repeatability. In addition, subcutaneous osteogenesis is superficial and can be easily detected by ultrasonic wave.

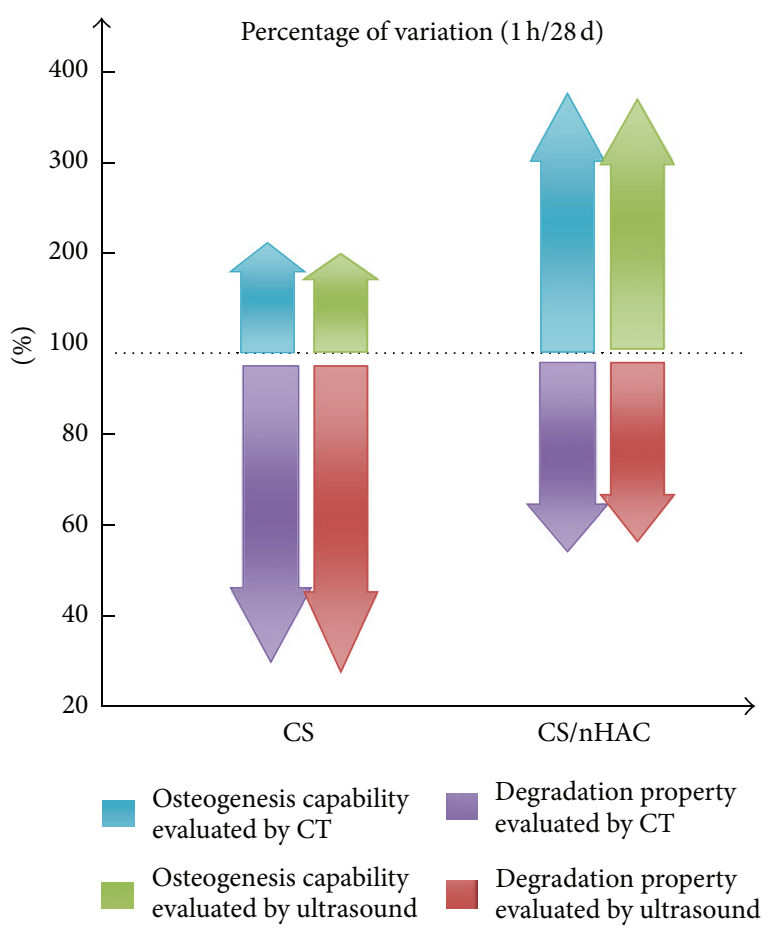

FIgURE 4: The percentage variation $(1 \mathrm{~h} / 28 \mathrm{~d})$ of osteogenesis capability (up the $100 \%$ horizontal ordinate, the CT value, and ultrasound $\mathrm{GV}$ ratio of $1 \mathrm{~h} / 28 \mathrm{~d}$ ) and degradation property (under the $100 \%$ horizontal ordinate, CT mask volume, and ultrasound section pixel of $1 \mathrm{~h} / 28 \mathrm{~d}$ ) via CT and ultrasound (data from Table 3 ). As same as CT, ultrasound shows that the osteogenesis capability and degradation property are better in CS/nHAC than in CS.

Ultrasound shows wide prospects in tissue engineering. We could alternatively adopt 3D ultrasound to count mean volume gray and thus to improve the accuracy of ultrasound evaluation, and a sole evaluation index for elastic property could be explored by ultrasonic elastosongraphy. Potential limitations also reside in our study. Although the same principles are utilized by different ultrasound devices, comparison and transformation of experimental data among devices would be disturbed by the differences in monitoring sites, measured parameters, and measurement precisions. Besides, ultrasound is not sufficiently accurate in the assessment of deep bone defect repair due to the significant interference of the surrounding tissues.

\section{Conclusions}

The evaluation results by the two imaging techniques, ultrasound and CT, showed a fairly significant consistency. Ultrasound is a convenient method for biomaterial assessment and provides a novel index of bone density by mean grey value, which reflects osteogenesis capability as well as CT value. Pixel analysis of ultrasound images is also a useful parameter in assessment of degradation property of the injectable biomaterials, which is correlated to CT. Our findings emphasize the feasibility of ultrasound in evaluation of biomaterials. Ultrasound has a bright future for application and evaluation 
TABLE 4: The $1 \mathrm{~h} / 28 \mathrm{~d}$ ratios of CT value and ultrasound GV and of CT mask volume and ultrasound section pixel. Both CT and ultrasound are approximate to evaluate osteogenesis capability and degradation property, and CS/nHAC is more suitable for bone tissue engineering than CS.

\begin{tabular}{lcccc}
\hline \multirow{2}{*}{ Group } & \multicolumn{2}{c}{ Percentage variation $(1 \mathrm{~h} / 28 \mathrm{~d})$} & Degradation property \\
& $\mathrm{CT} \mathrm{HU}_{m}$ & Ultrasound GV & CT volume & Ultrasound section area \\
\hline $\mathrm{CS}$ & $214.13 \%$ & $187.33 \%$ & $35.36 \%$ & $30.93 \%$ \\
$\mathrm{CS} / \mathrm{nHAC}$ & $381.18 \%$ & $368.43 \%$ & $55.34 \%$ & $57.45 \%$ \\
$P$ value & 0.009 & 0.000 & 0.030 & 0.008 \\
\hline
\end{tabular}

of bone tissue engineering. Additional research is needed before it is widely use.

\section{Conflict of Interests}

The authors declare that they have no direct financial relations with the commercial identities mentioned in this paper that might lead to a conflict of interests for any of the authors.

\section{Authors' Contribution}

Yan Chen and Yuting Yan have contributed equally to this paper.

\section{Acknowledgments}

The authors are grateful for the financial support from the National Basic Research Program of China (Program 973, no. 2011CB710901), National Natural Science Foundation of China (nos. 81101348 and 31000431), Natural Science Foundation of Guangdong Province, China (nos. 10451051501004727 and 10151051501000107), Guangdong Medical Scientific Research Project (A2013385), Doctoral Fund of Ministry of Education, China (20114433120004), the Beijing Nova Program (no. 2010B011), Program for New Century Excellent Talents (NCET) in University from Ministry of Education of China, National Undergraduate Training Programs for Innovation and Entrepreneurship, China (no. 201212121020), and outstanding person fund of Zhujiang Hospital.

\section{References}

[1] B. Jeong, Y. H. Bae, D. S. Lee, and S. W. Kim, "Biodegradable block copolymers as injectable drug-delivery systems," Nature, vol. 388 , no. 6645 , pp. 860-862, 1997.

[2] M. K. Nguyen and D. S. Lee, "Injectable biodegradable hydrogels," Macromolecular Bioscience, vol. 10, no. 6, pp. 563-579, 2010.

[3] A. A. Amini and L. S. Nair, "Injectable hydrogels for bone and cartilage repair," Biomedical Materials, vol. 7, no. 2, Article ID 024105, 2012.

[4] X. Li, L. Wang, Y. Fan, Q. Feng, F. Cui, and F. Watari, "Nanostructured scaffolds for bone tissue engineering," Journal of Biomedical Materials Research A, vol. 101, no. 8, pp. 24242435, 2013.
[5] X. Li, Y. Yang, Y. Fan, Q. Feng, F. Cui, and F. Watari, "Biocomposites reinforced by fibers or tubes, as scaffolds for tissue engineering or regenerative medicine," Journal of Biomedical Materials Research A, 2013.

[6] G. Hulsart-Billstrom, K. Bergman, B. Andersson, J. Hilborn, S. Larsson, and K. B. Jonsson, "A uni-cortical femoral defect model in the rat: evaluation using injectable hyaluronan hydrogel as a carrier for bone morphogenetic protein-2," Journal of Tissue Engineering and Regenerative Medicine, 2012.

[7] X. Li, C. A. van Blitterswijk, Q. Feng, F. Cui, and F. Watari, “The effect of calcium phosphate microstructure on bone-related cells in vitro," Biomaterials, vol. 29, no. 23, pp. 3306-3316, 2008.

[8] X. Li, H. Liu, X. Niu et al., "The use of carbon nanotubes to induce osteogenic differentiation of human adipose-derived MSCs in vitro and ectopic bone formation in vivo," Biomaterials, vol. 33, no. 19, pp. 4818-4827, 2012.

[9] H.-T. Liao, C.-T. Chen, and J.-P. Chen, "Osteogenic differentiation and ectopic bone formation of canine bone marrow-derived mesenchymal stem cells in injectable thermoresponsive polymer hydrogel," Tissue Engineering C: Methods, vol. 17, no. 11, pp. 1139-1149, 2011.

[10] H. Zhou and H. H. K. Xu, "The fast release of stem cells from alginate-fibrin microbeads in injectable scaffolds for bone tissue engineering," Biomaterials, vol. 32, no. 30, pp. 7503-7513, 2011.

[11] S. Jo, S. Kim, T. H. Cho, E. Shin, S. J. Hwang, and I. Noh, "Effects of recombinant human bone morphogenic protein2 and human bone marrow-derived stromal cells on in vivo bone regeneration of chitosan-poly(ethylene oxide) hydrogel," Journal of Biomedical Materials Research A, vol. 101, no. 3, pp. 892-901, 2013.

[12] Y. Chen, Z. Huang, X. Li et al., "In vitro biocompatibility and osteoblast differentiation of an injectable chitosan/nanohydroxyapatite/collagen biomaterial," Journal of Nanomaterials, vol. 2012, Article ID 401084, 6 pages, 2012.

[13] Y. Chen, S. Li, X. Li et al., "Noninvasive evaluation of injectable chitosan/nano-hydroxyapatite/collagen biomaterial via ultrasound," Journal of Nanomaterials, vol. 2012, Article ID 939821, 7 pages, 2012.

[14] Y. Zhang, W. Fan, L. Nothdurft et al., "In vitro and in vivo evaluation of adenovirus combined silk fibroin scaffolds for bone morphogenetic protein-7 gene delivery," Tissue Engineering C: Methods, vol. 17, no. 8, pp. 789-797, 2011.

[15] N. M. Mueske, L. S. Chan, and T. A. Wren, "Reliability of lateral distal femur dual-energy x-ray absorptiometry measures," Journal of Clinical Densitometry, pp. S1094-S6950, 2013.

[16] T. Whitmarsh, K. D. Fritscher, L. Humbert et al., "Hip fracture discrimination from dual-energy $\mathrm{x}$-ray absorptiometry by statistical model registration," Bone, vol. 51, no. 5, pp. 896-901, 2012. 
[17] J. Bacchetta, K. Wesseling-Perry, V. Gilsanz, B. Gales, R. C. Pereira, and I. B. Salusky, "Idiopathic juvenile osteoporosis: a cross-sectional single-centre experience with bone histomorphometry and quantitative computed tomography," Pediatric Rheumatology Online Journal, vol. 11, no. 1, article 6, 2013.

[18] K.-T. Khaw, J. Reeve, R. Luben et al., "Prediction of total and hip fracture risk in men and women by quantitative ultrasound of the calcaneus: EPIC-Norfolk prospective population study," The Lancet, vol. 363, no. 9404, pp. 197-202, 2004.

[19] W. P. Olszynski, J. P. Brown, J. D. Adachi, D. A. Hanley, G. Ioannidis, and K. S. Davison, "Multisite quantitative ultrasound for the prediction of fractures over five years of follow-up: the canadian multicentre osteoporosis study, Journal of Bone and Mineral Research, 2013.

[20] K. Kim, C. G. Jeong, and S. J. Hollister, "Non-invasive monitoring of tissue scaffold degradation using ultrasound elasticity imaging," Acta Biomaterialia, vol. 4, no. 4, pp. 783-790, 2008.

[21] C. M. Langton, S. B. Palmer, and R. W. Porter, "The measurement of broadband ultrasound attenuation in cencellous bone," Engineering in Medicine, vol. 13, no. 2, pp. 89-91, 1984.

[22] S. Nayak, I. Olkin, H. Liu et al., "Meta-analysis: accuracy of quantitative ultrasound for identifying patients with osteoporosis," Annals of Internal Medicine, vol. 144, no. 11, pp. 832-841, 2006.

[23] M. H. Lu, R. Mao, Y. Lu, Z. Liu, T. F. Wang, and S. P. Chen, "Quantitative imaging of young's modulus of soft tissues from ultrasound water jet indentation: a finite element study," Computational and Mathematical Methods in Medicine, vol. 2012, Article ID 979847, 6 pages, 2012.

[24] S. Kreitz, G. Dohmen, S. Hasken, T. Schmitz-Rode, P. Mela, and S. Jockenhoevel, "Nondestructive method to evaluate the collagen content of fibrin-based tissue engineered structures via ultrasound," Tissue Engineering C: Methods, vol. 17, no. 10, pp. 1021-1026, 2011.

[25] W. R. Taylor, E. Roland, H. Ploeg et al., "Determination of orthotropic bone elastic constants using FEA and modal analysis," Journal of Biomechanics, vol. 35, no. 6, pp. 767-773, 2002.

[26] B. Kundu, R. Rajkhowa, S. C. Kundu, and X. Wang, "Silk fibroin biomaterials for tissue regenerations," Advanced Drug Delivery Reviews, vol. 65, no. 4, pp. 457-470, 2012.

[27] N. S. Binulal, A. Natarajan, D. Menon, V. K. Bhaskaran, U. Mony, and S. V. Nair, "Gelatin nanoparticles loaded poly(epsiloncaprolactone) nanofibrous semi-synthetic biomaterials for bone tissue engineering," Biomedical Materials, vol. 7, no. 6, article 65001, 2012.

[28] H. Wang, Q. Zou, O. C. Boerman et al., "Combined delivery of bmp-2 and bfgf from nanostructured colloidal gelatin gels and its effect on bone regeneration in vivo," Journal of Controlled Release, vol. 166, no. 2, pp. 172-181, 2013.

[29] K. Rezwan, Q. Z. Chen, J. J. Blaker, and A. R. Boccaccini, "Biodegradable and bioactive porous polymer/inorganic composite scaffolds for bone tissue engineering," Biomaterials, vol. 27, no. 18, pp. 3413-3431, 2006. 

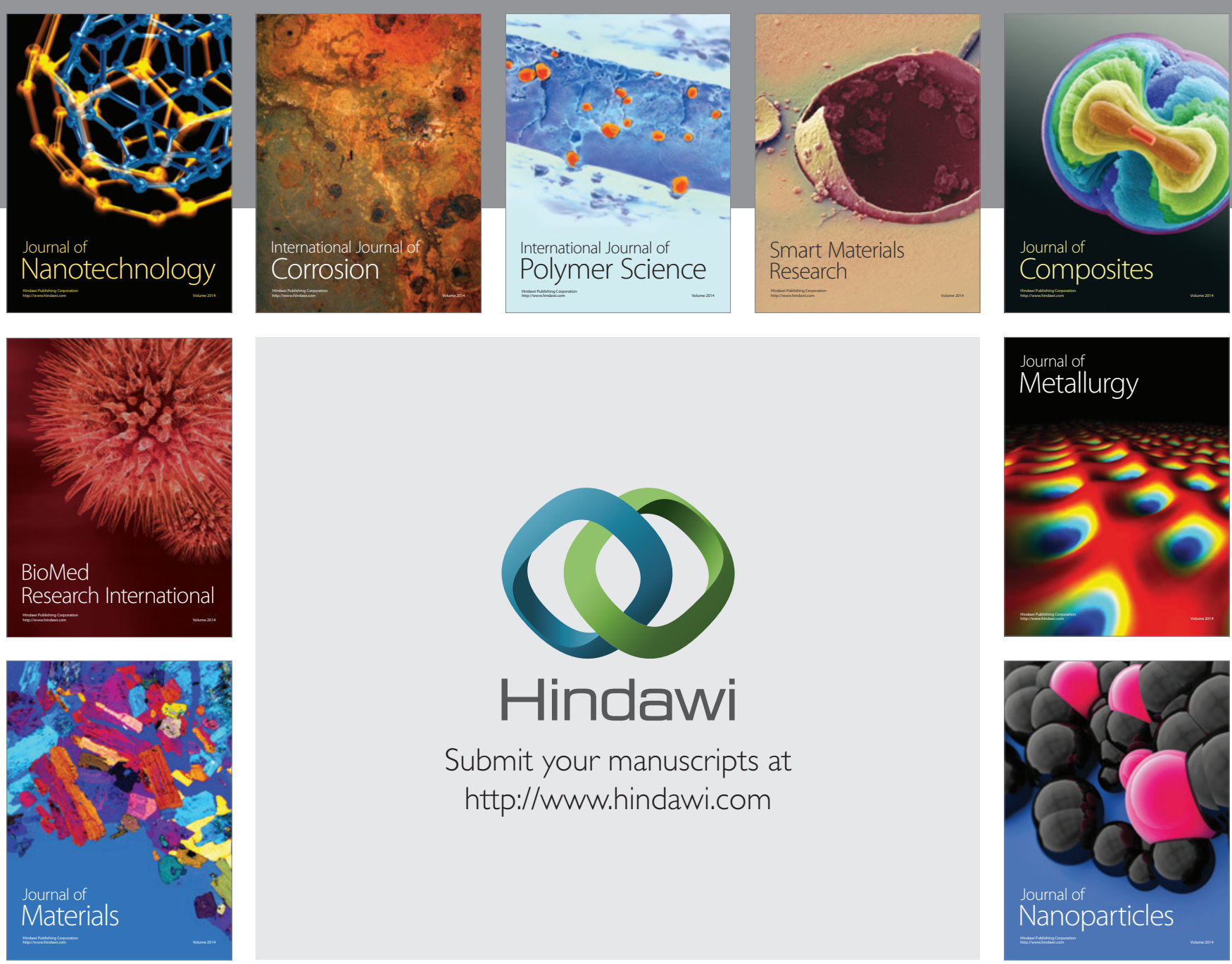

Submit your manuscripts at http://www.hindawi.com
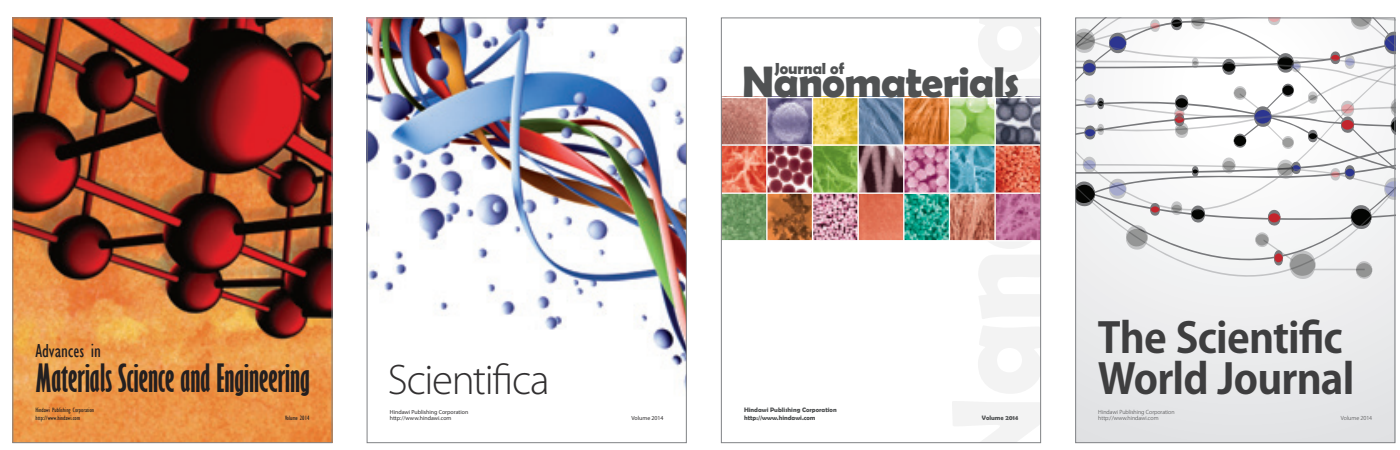

\section{The Scientific World Journal}
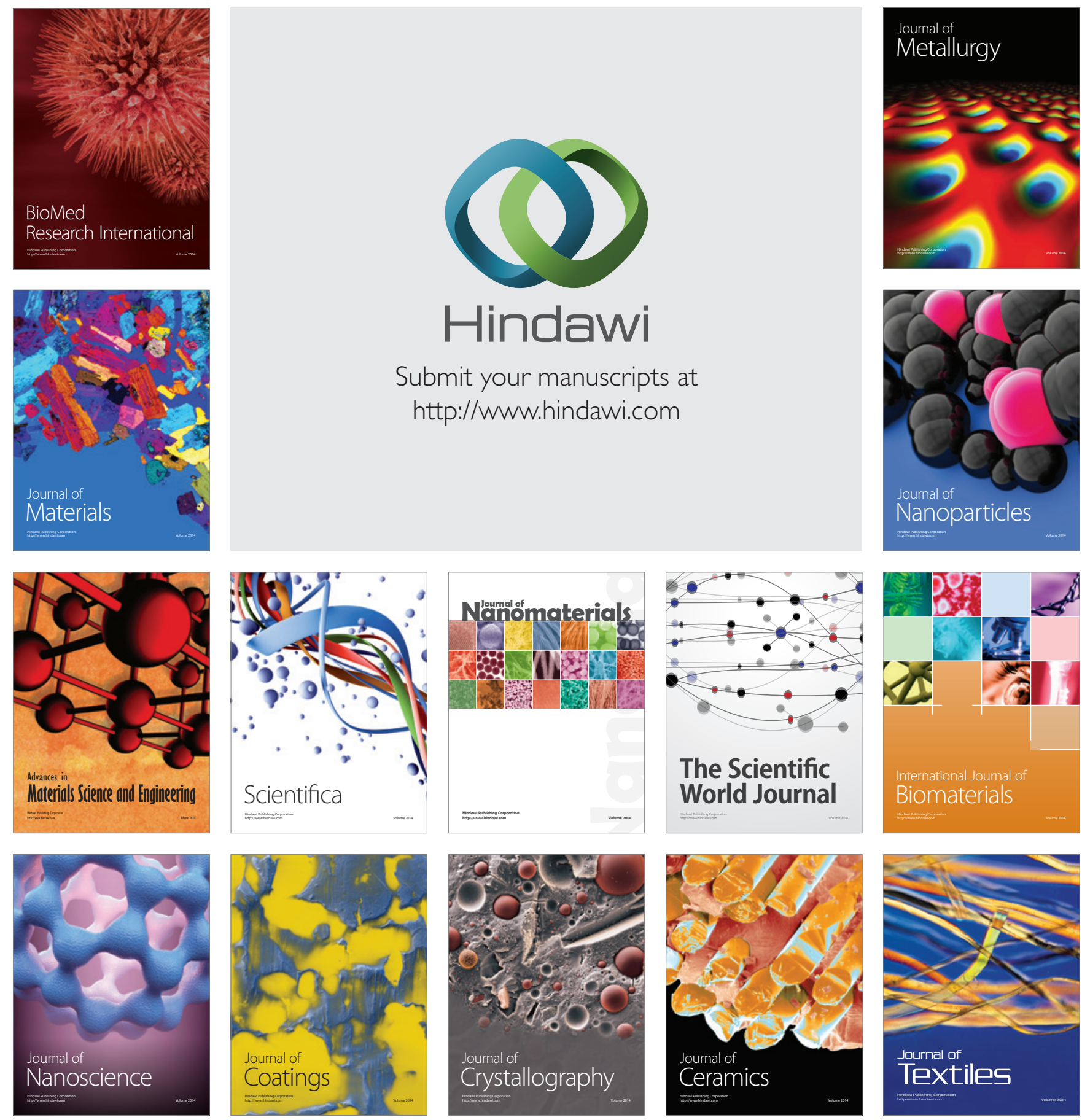